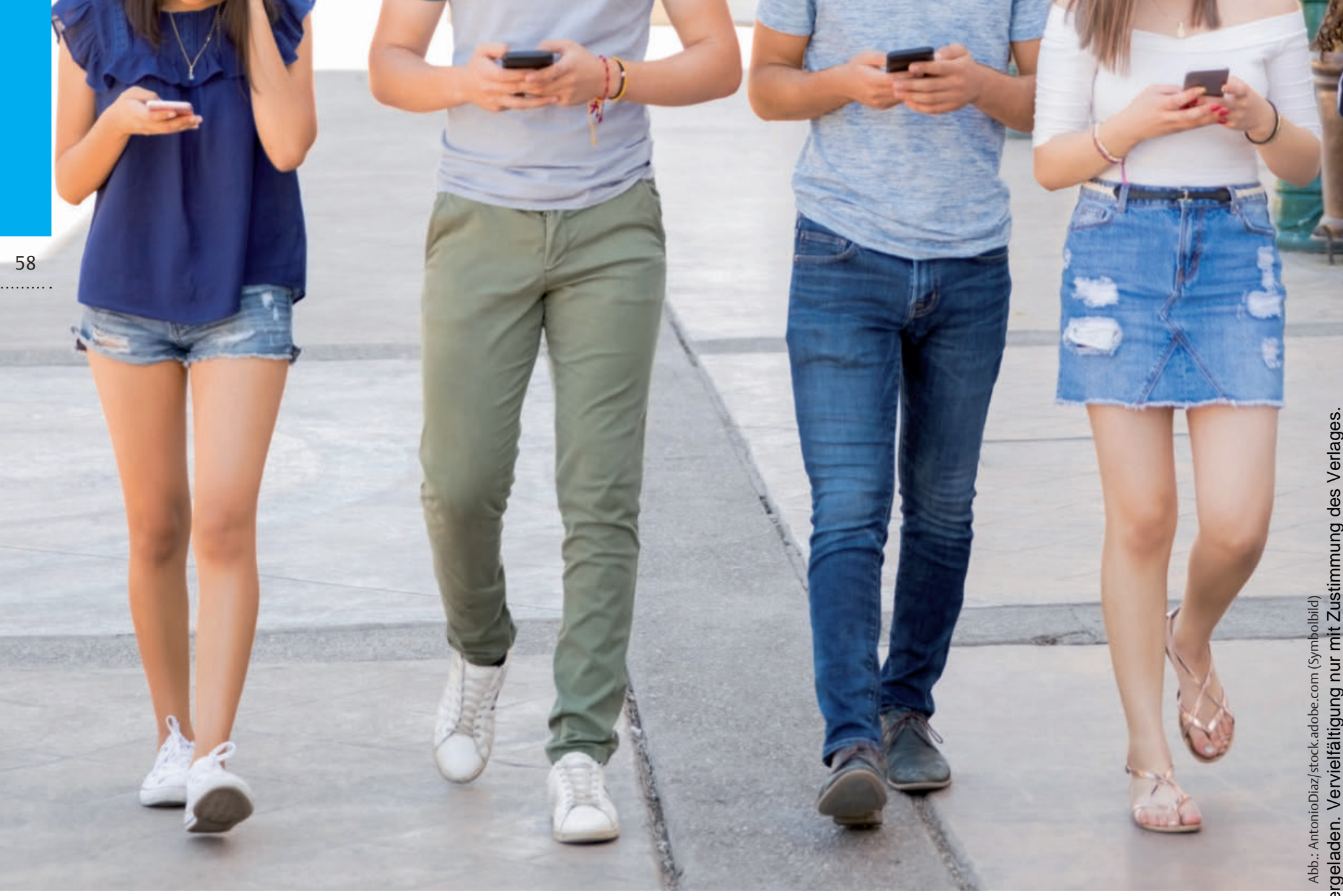

\title{
Jede Generation hat eigene Werte
}

Generation Z Veränderungen finden ständig statt - egal, ob in der Politik, Mode oder Technik. Seit ein bis zwei Jahren macht besonders eine Entwicklung Schlagzeilen: die viel diskutierte, manchmal belächelte und oft kritisierte Generation Z. Mit ihren Werten und Einstellungen stellt sie Arbeitgeber, Kollegen und Lehrende vor neue Herausforderungen.

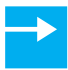

Umgangssprachlich ist eine Generation eine Summe von Personen, die zur selben Altersgruppe gehören. Die Soziologie definiert sie als „die Gesamtheit von Menschen ungefähr gleicher Altersstufe mit ähnlicher sozialer Orientierung und einer Lebensauffassung, die ihre Wurzeln in den prägenden Jahren einer Person hat" [1]. Im Generationenkonzept nach Howe und Strauss sind bisher 19 Generationen lokalisiert, die in der Zeit von 1588-1617 (Puritaner) beginnen und bis heute reichen [2].

Die aktuellen Generationen $\rightarrow$ Zurzeit existieren vor allem vier Generationen: die Babyboomer sowie Angehörige der Generationen X, $Y$ und $Z$ (॰ TAB. 1) [1]. Jede von ihnen hat ihre
Prägungen, Stärken und Schwächen. Es gilt sie zu verstehen, wobei dieses Verstehen in alle Richtungen gehen sollte: So ist es nötig, dass sich etwa die Babyboomer und alle anderen Generationen in der gleichen Form mit der Generation $Z$ auseinandersetzen wie die Generation $Z$ mit den vorangegangenen Generationen [3].

Entwickler von Generationenkonzepten argumentieren, dass Generationen nicht nur aufgrund von Zeitspannen ihre Namen bekommen, sondern es auch die historischen Ereignisse sind, die sie prägen. Problematisch wäre es, das Konzept der Generation zu eng zu sehen, denn es kommt nicht vorrangig auf das Geburtsjahr an. Für jede Generation gibt es Werte, die sie besonders dominieren und Privatleben,
Berufstätigkeit sowie die Rolle als Konsument und Medienschaffender beeinflussen [3]. Die Prägung und Einflüsse aus der Jugend bleiben nach Ansicht der Forscher über das ganze Leben weitestgehend konstant [2]. Dennoch kann man bei allen bislang existierenden Generationenkonzepten nicht von einer Homogenität ausgehen. Es besteht immer eine Varianz, die aber nichts an den tendenziellen Unterschieden und Prägungen der Generationen ändert, die für sie charakteristisch sind (॰ TAB. 2, S. 60) [1, 3].

Digital Natives $\rightarrow$ Publikationen wie „Digital Cowboys“ oder „The Year without Pants“ treffen Aussagen dazu, wie die heutigen Jugendlichen (vorwiegend der Generation Z) als Digital 
Natives agieren [4, 5]. Sie sind mit dem Internet aufgewachsen und besitzen eine Technologievertrautheit. Sie wissen, wie man mit einem Smartphone gute Fotos schießt, den FacebookAccount bearbeitet und Likes setzt. Sie twittern, laden Bilder und Videos auf „Insta“ hoch, checken nebenbei Mails und teilen allen auf WhatsApp mit, dass sie sich gerade eine Pizza bestellt haben oder irgendwo im Café sitzen. Hinzu kommt eine Unbekümmertheit im Umgang mit der Informationstechnologie: „Wo ein Babyboomer noch zaudert, um nicht etwas falsch zu machen, drückt der Digital Native einfach auf den Knopf“" [3]. Sie besitzen die Bereitschaft, sich in gläserne Menschen zu verwandeln, und leben sehr real in einer digitalen Welt.

Rund um die Uhr online $\rightarrow$ Digital Natives sind immer online, immer informiert und haben eine klare Botschaft, die Arbeitgeber generell gerne hören: „Früher war der Nine-to-five-Job die Regel; heute setzt sich immer mehr 24/7 durch - also die ständige Bereitschaft“ [6]. Doch die Generation Z stellt auch ihre eigenen Bedingungen. Sie sieht, anders als die Babyboomer, nicht die Notwendigkeit einer 24/7-Dienstleistung. Sie lässt sich auch nicht wie die Generation Y den Verzicht auf Selbstbestimmung und Flexibilität durch Geld abgelten. Sie zieht eine klare Linie zwischen Arbeit und Privatem. 24/7 bezieht sich demnach eher auf die Tatsache, immer präsent und online zu sein, um andere User mit neuen Infos zu befriedigen, damit sie immer wieder auf die Person aufmerksam werden und diese nicht vergessen [7]. Der ideale Arbeitsplatz soll flexibel sein, die Arbeitsflächenstrukturierung mündet in der Papierlosigkeit, die Arbeitsflächenoptimierung heißt Desk-Sharing und die Arbeitsflächenminimierung Homeoffice.

Generation Z als Arbeitnehmer $\rightarrow$ Zur Generation Z gehören diejenigen, die entweder gerade eine Ausbildung absolvieren, studieren oder auf den Arbeitsmarkt drängen. Im Magazin Wirtschaftswoche war zu lesen: „Die Absolventen und Jobeinsteiger können es sich leisten, auf Traditionen zu pfeifen. Kein Arbeitgeber kann es sich in Zeiten des Fachkräftemangels und des demografischen Wandels noch rausnehmen, ihre Wünsche und Bedürfnisse zu ignorieren“ " 8]. Auch Vertreter der Hochschulen argumentieren in diese Richtung und betonen: „Die können ihr Ding durchziehen, weil die demografischen Veränderungen ihre Wett-

\begin{tabular}{lll} 
Generationen in Deutschland & Geburtsjahre & prägende Jahre \\
\hline Babyboomer (ca. 20,7 Mio.) & $1946-1964$ & $1957-1979$ \\
\hline Generation X (ca. 17,8 Mio.) & $1965-1979$ & $1976-1994$ \\
\hline Generation Y (ca. 14,8 Mio.) & $1980-1995$ & $1991-2010$ \\
\hline Generation Z (ca. 14,7 Mio.) & $1996-?$ & $2007-?$ \\
\hline
\end{tabular}

TAB. 1 Die vier aktuellen Generationen Deutschlands

bewerbssituation auf dem Arbeitsmarkt durchschlagend verbessern" [9].

Auch die 22-jährige Berufseinsteigerin Laura gehört zur Generation Z. Sie hat im September 2019 ihr Staatsexamen zur Physiotherapeutin abgeschlossen. Einen Job in einer Praxis zu finden war für sie nicht schwer. Aufgrund des Fachkräftemangels hatte sie den 20-StundenArbeitsvertrag schon vor ihrer eigentlichen Berufsanerkennung in der Tasche. Sie hat ihrer neuen Arbeitgeberin aber gleich klargemacht, dass sie erst ab Mai 2020 einsteigen kann, da sie vorher noch nach Australien möchte, um sich dort von den Strapazen des Examens zu erholen. Mehr als 20 Stunden pro Woche möchte sie sowieso nicht arbeiten, da sie ab Februar 2020 auch noch berufsbegleitend studiert und es ihr sonst zu viel wird. Auf die Nachfrage ihrer neuen Chefin, wie sie denn studieren kann, obwohl sie sich in Australien befindet, äußert Laura lediglich, dass es viele Online-Module gibt, zu denen sie sich von überall aus zuschalten kann. Solange sie ihre Hausarbeiten abgibt, wäre doch alles prima.

Man kann ja alles googeln $\rightarrow$ Überstunden möchte Laura nur ungern machen, da sie auf ein Verschmelzen von Arbeits- und Pri-

vatwelt ganz klar verzichten möchte. Deswegen sind konstante Arbeitszeiten wichtig für sie. Die 22-Jährige hat sich deshalb auch schon ein zweites Smartphone zugelegt, da sie im privaten Bereich natürlich immer „on“ sein möchte, dies für den beruflichen Bereich aber ablehnt. Zu Hause eventuell noch etwas nachzuarbeiten kommt für sie nicht in Frage. Wenn sie Informationen zu einem Krankheitsbild oder zu evidenz-basierten Behandlungsmethoden benötigt, kann sie das ja auch in der

Praxis mit ihrem Smartphone googeln. Eine eigene kleine Büroecke in der Praxis wäre cool, und Laura hat sich schon jetzt in Gedanken mit vielen Details der Einrichtung beschäftigt: farbenfrohe Kleinigkeiten auf dem Schreibtisch, ein bequemer Stuhl und Fotos an den Wänden.

Hausbesuche möchte Laura nur machen, wenn dies mit dem Fahrrad oder mit einem Praxisfahrzeug möglich ist. Sie hat bisher selten ein Auto gebraucht und wenn sie doch eines benötigt, gibt es Car-Sharing. Eigentlich ist es ja sowieso schöner, in der Praxis zu arbeiten und so häufiger mit Kollegen ins Gespräch zu kommen. Auf die Frage hin, ob Laura denn von einem Gehalt für 20 Stunden leben kann, antwortet sie, dass sie sowieso erst einmal bei den Eltern wohnen wird, und findet das „supi“ so.

\section{Verantwortung übernehmen: Wie soll das} gehen? $\rightarrow$ Dass Laura von ihrer neuen Arbeitgeberin Fortbildungen bezahlt bekommt, rechnet sie ihr hoch an. Jedoch wünscht sie sich ein breites Spektrum an Fortbildungsmöglichkeiten und möchte sich selbst für die beste Kombination entscheiden, unabhängig davon, ob diese dem Unternehmensinteresse dient. Es muss ihr ja schließlich auch Spaß machen.

Bezogen auf ihr Studium macht sich Laura schon jetzt Gedanken, denn gleich im ersten Semes-

\section{Smombies}

(„Smartphone-Zombies“) nehmen durch den ständigen Blick aufs Handy ihre Umgebung kaum noch wahr. Das kann zum

Beispiel im Straßenverkehr schnell gefährlich werden.

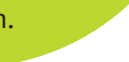
ter muss sie die Verantwortung für eine Projektarbeit übernehmen. Selber nachdenken, was zu tun ist, und sich die Arbeit so einteilen, dass das Projekt rechtzeitig fertig wird

- wie soll das gehen? Sie hat dafür nur drei Monate Zeit und überhaupt keine Ahnung, was zu tun ist. Sie hat aber schon einen Plan B: Ein Freund von ihr kennt sich sehr gut mit der Thematik aus, und sie wird ihn einfach fragen, ob er das nicht für sie erledigen kann. 


\begin{tabular}{|c|c|c|c|c|}
\hline & Babyboomer & Generation X & Generation Y & Generation Z \\
\hline $\begin{array}{l}\text { prägende } \\
\text { Einflüsse }\end{array}$ & $\begin{array}{l}\text { Wirtschaftswunder, Mauerbau, } \\
\text { Kubakrise, Ermordung John F. } \\
\text { Kennedy, Woodstock, } \\
\text { Mondlandung, TV, Familienbild }\end{array}$ & $\begin{array}{l}\text { Ölkrise, RAF, Tschernobyl, } \\
\text { Space Shuttle Challenger, } \\
\text { Mauerfall, Atari, Walkman, } \\
\text { Video, MTV, Scheidungsraten }\end{array}$ & $\begin{array}{l}\text { Globalisierung, Klimawandel, } \\
\text { Golfkrieg, 9/11, Osama bin } \\
\text { Laden, Euro, Tsunami, Hurrikan } \\
\text { Katrina, Facebook, Handy, } \\
\text { „Helikopter“-Eltern }\end{array}$ & $\begin{array}{l}\text { Finanz- und Wirtschaftskrise, } \\
\text { Erdbeben in Haiti, Fukushima, } \\
\text { IS, Reality-TV, iPad, } \\
\text { Smartphone, Bologna-Reform, } \\
\text { „Kronprinz-Kindheit“ }\end{array}$ \\
\hline $\begin{array}{l}\text { typische } \\
\text { Eigenschaften }\end{array}$ & $\begin{array}{l}\text { optimistisch, tatkräftig, } \\
\text { teamorientiert, konfliktscheu, } \\
\text { pflichtbewusst }\end{array}$ & $\begin{array}{l}\text { skeptisch, pragmatisch, } \\
\text { eigenständig, direkt, } \\
\text { pflichtergeben }\end{array}$ & $\begin{array}{l}\text { authentisch, sprunghaft, } \\
\text { sozial vernetzt, anspruchsvoll, } \\
\text { selbstbewusst }\end{array}$ & $\begin{array}{l}\text { realistisch, flüchtig, } \\
\text { hypervernetzt, fordernd, } \\
\text { egozentrisch }\end{array}$ \\
\hline Werte & $\begin{array}{l}\text { Demokratie, Gemeinschaft, } \\
\text { Entscheidungsfreiheit, } \\
\text { Idealismus, Konsens, Loyalität, } \\
\text { Ordnung, Status, Sorgfalt, } \\
\text { Strebsamkeit }\end{array}$ & $\begin{array}{l}\text { Autonomie, Erfolg, Flexibilität, } \\
\text { Gegenleistung, Individualismus, } \\
\text { Kompetenz, Produktivität, } \\
\text { Professionalität, Vielfalt, } \\
\text { Zielorientierung }\end{array}$ & $\begin{array}{l}\text { Abwechslung, Beteiligung, } \\
\text { Lifestyle, Nachhaltigkeit, } \\
\text { Selbstverwirklichung, } \\
\text { Sinnstiftung, Spaß, Transparenz, } \\
\text { Zugehörigkeit, Zusammen- } \\
\text { arbeit }\end{array}$ & $\begin{array}{l}\text { Erfüllung, Informationsfreiheit, } \\
\text { Integrität, Sicherheit, Sparsam- } \\
\text { keit, Stabilität, Unternehmer- } \\
\text { geist, Unverbindlichkeit, } \\
\text { Vernetzung, Zweckmäßigkeit }\end{array}$ \\
\hline Arbeitsethos & $\begin{array}{l}\text { Babyboomer haben eine hohe } \\
\text { Arbeitsmoral und wollen eine } \\
\text { „Bilderbuchkarriere“ hinlegen, } \\
\text { um den vermeintlichen } \\
\text { Erwartungen der Gesellschaft } \\
\text { zu entsprechen. Sie sind } \\
\text { intrinsisch motiviert, qualitativ } \\
\text { hochwertige Arbeit zu leisten. }\end{array}$ & $\begin{array}{l}\text { Produktivität ist das Wichtigste } \\
\text { für Xler. Sie konzentrieren sich } \\
\text { auf Ergebnisse und das } \\
\text { Endresultat. Unternehmensziele } \\
\text { sind wichtiger als persönliche } \\
\text { Ziele. Sie tun, „was nötig ist“, } \\
\text { um einen Job zu erledigen, } \\
\text { erwarten aber auch, dafür } \\
\text { belohnt zu werden. }\end{array}$ & $\begin{array}{l}\text { Abkehr von der kompletten } \\
\text { Hingabe und Aufopferung für } \\
\text { die Arbeit. Yler sind fixiert auf } \\
\text { persönliche Lebensziele und } \\
\text { Sinnfindung. Sie machen einen } \\
\text { Job, solange er der eigenen } \\
\text { Selbstverwirklichung dient, } \\
\text { anderenfalls suchen sie sich } \\
\text { etwas Neues. }\end{array}$ & $\begin{array}{l}\text { Zler wollen vor allem die } \\
\text { hohen Erwartungen der Eltern } \\
\text { erfüllen. Sie sind noch auf der } \\
\text { Suche nach dem eigenen } \\
\text { Antrieb fürs Berufsleben, } \\
\text { wünschen sich aber einen } \\
\text { sicheren Arbeitsplatz und } \\
\text { wollen einen Beruf, der sie } \\
\text { erfüllt und Spaß macht. }\end{array}$ \\
\hline Hauptmerkmal & Selbsterfüllung & Perspektivenlosigkeit & Leistungsbereitschaft & Flatterhaftigkeit \\
\hline
\end{tabular}

TAB. 2 Typische Werte und Eigenschaften der vier aktuellen Generationen

Typische Merkmale $\rightarrow$ Das Fallbeispiel zeigt die in $\odot$ TAB. 2 dargestellten Charakteristika der Generation Z in ihren möglichen Auswirkungen auf die physiotherapeutische Praxis:

$\rightarrow$ Die Generation Z will ihre Privatsphäre unter allen Umständen schützen.

$\rightarrow$ Sie bestehen auf geregelte Arbeitszeiten.

$\rightarrow$ Homeoffice als Arbeitsplatz ist generell out. Die Generation Z beansprucht zwar das Anrecht darauf, versteht dies aber als Möglichkeit, nach Lust und Laune zu Hause zu bleiben und von dort aus in Ruhe zu arbeiten. In einer Praxis ist die Diskussion um Homeoffice weniger relevant. In der Ausbildung zeigen sich jedoch Tendenzen, dass Auszubildende es bevorzugen, Arbeitsaufträge, die sie auch zu Hause machen könnten, lieber in einer Kleingruppe mit geteilter Verantwortung in der Schule erledigen.

$\rightarrow$ Die physiotherapeutische Praxis und die Schule fungieren als Zweitwohnung, klar abgetrennt vom privaten Lebensbereich, aber trotzdem wohnlich und kuschelig.

$\rightarrow$ „Don't manage me“ gilt als ultimative Forderung. Eine Studie zeigt, dass die Generation Z nicht von älteren Generationen ver- plant, verändert oder entwickelt werden möchte [10]. Sie entscheidet selbst, was sie hinsichtlich ihrer Qualifizierung macht, wann sie es macht und wie. Die Realität zeigt, dass sie sich damit häufig selbst überschätzt und erforderliche Qualifikationen oft (noch) nicht vorhanden sind [10]. Für Arbeitgeber oder Lehrende bedeutet dies, der Generation Z zu vermitteln, dass hinter „don't manage me“ viel Selbstverantwortung und Eigeninitiative stecken. Problematisch ist, dass sich die Generation Z als hoch qualifiziert einstuft und ein Scheitern aus ihrer Sicht Ausdruck falschen Führungsstils ist.

$\rightarrow$ Always on und always off: Die Generation Z praktiziert privat zwar ein „Always on“, lehnt dies aber im Beruf ab. Private Kommunikation sollte auf Notfälle oder Pausen begrenzt sein. Denkt man an die Allgegenwärtigkeit von Smartphones, auch im Unterricht, wird deutlich, wie schwer die Durchsetzung einer entsprechenden Regel ist.

Mit anderen Generationen auseinandersetzen $\rightarrow$ Durch die aktuelle berufspolitische Situation, in der es mehr denn je gilt, die Evidenz der Physiotherapie zu erbringen, Berufsidentität zu erlangen und sich von anderen Professionen abzugrenzen, benötigen wir qualifizierte, professionelle und tatkräftige Personen, die bereit sind, Führungsverantwortung zu übernehmen. Die Herausforderung für die älteren Generationen wird es sein, sich mit dem Wertemuster der Generation Z auseinanderzusetzen und sie zu unterstützen, Selbstverantwortung und Verantwortung für die Profession zu übernehmen.

Die Generation Z muss ihr eigenes Sinnsystem verstehen und es kommunizieren. Für Praxen, Schulen und Hochschulen ist es wichtig, sich mit allen Generationen auseinanderzusetzen, deren Eigenschaften, Werte und Hauptmerkmale zu verstehen und die positiven Attribute für sich zu nutzen. Was auf keinen Fall funktioniert, ist diese individuellen Werte verändern zu wollen. Es liegt auf der Hand, dass man alle vier Generationen mit ihrem jeweiligen Sinnverständnis akzeptieren und unterschiedlich führen muss. Claudia Klein, Bachelor OT (NI)

$\mathbf{a}$ Literaturverzeichnis www.thieme-connect.de/products/physiopraxis > „Ausgabe 1/20“ 